OPEN ACCESS

Edited by:

Syed Nasir Abbas Bukhari, Al Jouf University, Saudi Arabia

Reviewed by: Qi Shan,

Chinese Academy of Fishery Sciences (CAFS), China

Pritam Kaur Sidhu, Kansas State University, United States

*Correspondence: Huanzhong Ding hzding@scau.edu.cn

Specialty section: This article was submitted to Experimental Pharmacology and

Drug Discovery,

a section of the journal

Frontiers in Pharmacology

Received: 10 June 2019

Accepted: 26 August 2019 Published: 20 September 2019

Citation:

Zhang H, Mao C, Li J, Huang Z, Gu X, Shen X and Ding H (2019) Pharmacokinetic/Pharmacodynamic Integration of Doxycycline Against

Mycoplasma hyopneumoniae in an In Vitro Model.

Front. Pharmacol. 10:1088. doi: 10.3389/fphar.2019.01088

\section{Pharmacokinetic/Pharmacodynamic Integration of Doxycycline Against Mycoplasma hyopneumoniae in an In Vitro Model}

\author{
Huilin Zhang, Chunxiao Mao, Jinju Li, Zilong Huang, Xiaoyan Gu, Xiangguang Shen and \\ Huanzhong Ding*
}

Guangdong Key Laboratory for Veterinary Drug Development and Safety Evaluation, South China Agricultural University, Guangzhou, China

Doxycycline is a broad-spectrum antibacterial drug. It is used widely to treat diseases caused by Mycoplasma species. We investigated the antibacterial activity of doxycycline against the Mycoplasma hyopneumoniae strain ATCC25934. The minimum inhibitory concentration (MIC) of doxycycline against $M$. hyopneumoniae determined by a microdilution method was $0.125 \mu \mathrm{g} / \mathrm{ml}$. Static time-kill curves with constant drug concentrations (0-64 MIC) showed that a bacteriostatic effect occurred if the doxycycline concentration reached $4 \mathrm{MIC}$. Doxycycline produced a maximum antimycoplasmal effect (reduction of $2.76 \log _{10} \mathrm{CFU} / \mathrm{ml}$ ) at $64 \mathrm{MIC}$ within $48 \mathrm{~h}$. The effect of doxycycline against $M$. hyopneumoniae was analyzed by a sigmoid $E_{\max }$ model, and there was high correlation between the kill rate and doxycycline concentration $\left(R^{2}=0.986\right)$. A one-compartment open model with first-order absorption was adopted and was used to simulate doxycycline pharmacokinetics in porcine plasma. The dynamic time-concentration curve showed that the area under the curve at $24 \mathrm{~h}$ $\left(\mathrm{AUC}_{24} \mathrm{~h}\right)$ and $\mathrm{C}_{\max }$ (peak concentration) after each drug administration was 1.78-48.4 $\mu \mathrm{g}$ $\mathrm{h} / \mathrm{ml}$ and $0.16-3.41 \mu \mathrm{g} / \mathrm{ml}$, respectively. The reduction of $M$. hyopneumoniae $\left(\log _{10} \mathrm{CFU} /\right.$ $\mathrm{ml}$ ) for $1,2.5,5,7.5,10,15,20$, and $30 \mathrm{mg} / \mathrm{kg}$ body weight was $0.16,1.29,1.75,2.94$, $3.35,3.91,4.35$, and 5.77 , respectively, during the entire experiment, respectively. When the dose was $>10 \mathrm{mg} / \mathrm{kg}$ body weight, continuous administration for 3 days could achieve a bactericidal effect. The correlation coefficient of $\mathrm{AUC}_{24} \mathrm{~h} / \mathrm{MIC}, \mathrm{C}_{\max } / \mathrm{MIC}$, and \%T > MIC (the cumulative percentage of time over a 24-h period that the drug concentration exceeds the $\mathrm{MIC}$ ) with antibacterial effect was $0.917,0.923$, and 0.823, respectively. Doxycycline showed concentration-dependent activity, and the value of $\mathrm{AUC}_{24} \mathrm{~h} / \mathrm{MIC}$ and $C_{\max } / \mathrm{MIC}$ required to produce a drop of $1 \log _{10} \mathrm{CFU} / \mathrm{ml}$ was $164 \mathrm{~h}$ and 9.89 , respectively.

Keywords: Mycoplasma hyopneumoniae, doxycycline, in vitro model, pharmacokinetic, pharmacodynamic

\section{INTRODUCTION}

Mycoplasma hyopneumoniae is the primary etiologic agent of enzootic pneumonia and is widespread in pig populations (Rebaque et al., 2018). M. hyopneumoniae possesses a limited number of genes, low guanine and cytosine content and has simple metabolic pathways, so it cannot synthesize some essential compounds (Weber Sde et al., 2012). This microorganism is found primarily on 
the mucosal surface of the trachea, bronchi, and bronchioles. The immunity of pigs can be reduced significantly upon $M$. hyopneumoniae infection, which can induce infection by other pathogens. If $M$. hyopneumoniae is present with other pathogens, such as Actinobacillus pleuropneumoniae, Pasteurella multocida, Streptococcus suis, or Haemophilus parasuis, respiratory infections are aggravated and leads to porcine respiratory disease complex, which can cause considerable economic losses to the pig industry (Sibila et al., 2009).

As vaccine alone has limited antagonism to considerable variability of bacterial strain and has a lack of efficacy in stopping horizontal spread; therefore, antimicrobial chemotherapy is still necessary (Villarreal et al., 2011). M. hyopneumoniae is resistant to antimicrobial agents that interfere with the metabolism of folic acid and cell-wall synthesis, such as sulfonamides, trimethoprim, and the $\beta$-lactam class of antibiotics. Macrolides, tetracyclines, fluoroquinolones, lincosamides, and pleuromutilins are active agents against M. hyopneumoniae (Hannan et al., 1997; Maes et al., 2008).

Doxycycline is a tetracycline with good antimicrobial activity, strong penetration into tissue, and good oral bioavailability (Baert et al., 2000; De Mil et al., 2016; Li et al., 2016). Doxycycline binds to the decoding center of the small ribosomal subunit of the bacterium to inhibit protein synthesis. It is used for treatment of porcine respiratory diseases due to its broad-spectrum antibacterial activity. Doxycycline has been used widely for treatment of mycoplasmal pneumonia in pigs. There have been many studies on the pharmacokinetics (PK) of doxycycline not only in pigs but also in calves (Meijer et al., 1993), goats (Li et al., 2016), sheep (Castro et al., 2009; Castro Robles et al., 2012), broilers (Yang et al., 2018), cats (Hartmann et al., 2008), and dogs (Sebbag et al., 2018). The doxycycline concentration in pigs is best fitted to a one-compartmental model with first-order absorption (Bousquet et al., 1998).

Studies of treatment of pigs infected on a farm have indicated that doxycycline has significant therapeutic activity against mycoplasmal pneumonia (Wang et al., 2010; Liu, 2015). The nutritional and environmental conditions must be strict if M. hyopneumoniae is cultured in vitro, and isolating clinical strains is difficult. Limited information is available on the PK/ pharmacodynamic (PD) interactions of antibacterial agents against Mycoplasma species, including doxycycline. However, in recent years, development of an in vitro $\mathrm{PK} / \mathrm{PD}$ model has produced a robust method for correlation research (de la Pena et al., 2004; Budha et al., 2009; Nicasio et al., 2012). Mitchell et al. (2013) studied the antimicrobial activity of various agents against Mycoplasma mycoides subspecies mycoides small colony (MmmSC) in vitro. The dilution model they used was simulated by increasing the volume, which cannot mimic the continuous process of drug elimination. Zhang et al. (2016) evaluated antimicrobial activity against Mycoplasma gallisepticum using a simple in vitro dynamic model that could eliminate the agent through an outlet, and the initial antimicrobial concentrations corresponded to various multiples of the minimum inhibitory concentration (MIC).

We investigated the effect of doxycycline against $M$. hyopneumoniae based on PK/PD interactions. The model was based on the studies mentioned above with some necessary modifications: (i) we added an absorption chamber to mimic absorption in animals, and (ii) the design of the dose regimen was in accordance with clinical practice. We wanted to provide a reference for dose-regimen optimization which could achieve a cure for Mycoplasma pneumoniae infection and minimize the opportunity for antimicrobial resistance.

We pursued four objectives: (i) the MIC of doxycycline against M. hyopneumoniae strain ATCC25934 was determined; (ii) the in vitro time-kill curves for doxycycline against M. hyopneumoniae in a defined artificial medium were established and the relationship between the kill rate and drug concentrations were fitted to the $E_{\max }$ model (see below); (iii) the relationship between $\mathrm{PK} / \mathrm{PD}$ indices and the effect of doxycycline against $M$. hyopneumoniae were investigated in an in vitro dynamic PK/PD model; and (iv) a scientific dosing guidance of doxycycline against $M$. hyopneumoniae was provided based on $\mathrm{PK} / \mathrm{PD}$ modeling.

\section{MATERIALS AND METHODS}

\section{Materials}

The standard strain of M. hyopneumoniae (ATCC25934) was provided by the Chinese Veterinary Microorganism Culture Collection Center (Beijing, China). A doxycycline hyclate standard (>99\%) was obtained from the China Institute of Veterinary Drug Control (Beijing, China). The artificial medium base of M. hyopneumoniae was purchased from Qingdao Hope Biological Technology (Qingdao, China). Sterile pig serum was supplied by Guangzhou Ruite Biological Technology (Guangzhou, China). Cysteine and the reduced form of nicotinamide adenine dinucleotide were supplied by Guangzhou Prob Information Technology. The "raw" form of doxycycline (87.4\%) was provided by Guangdong Dahuanong Animal Health Products (Guangdong, China).

\section{MIC Determination}

A microdilution method was used to determine the MIC of doxycycline against M. hyopneumoniae strain ATCC25934, as described by Tanner et al. (1993) with modifications. The exponential-phase culture was diluted with culture medium to the desired inoculum size of $10^{6} \mathrm{CFU} / \mathrm{ml}$. The culture medium of M. hyopneumoniae contained artificial medium base, $20 \%$ pig serum, trace cysteine, and nicotinamide adenine dinucleotide. A series of concentrations of doxycycline was prepared by twofold dilution (final concentration was $0.001-0.25 \mu \mathrm{g} / \mathrm{ml}$ ) with media. Each of the first to the ninth column was added $0.1 \mathrm{ml}$ of media with different concentrations of doxycycline and $0.1 \mathrm{ml}$ of $M$. hyopneumoniae inoculum. A growth control (M. hyopneumoniae inoculum in the absence of antimicrobial agents), an end-point control (blank medium at $\mathrm{pH}$ 6.8) and a sterility control (sterility medium) were included. After sealing with a gas-permeable film, plates were cultured at $37^{\circ} \mathrm{C}$ in an atmosphere of $5 \% \mathrm{CO}_{2}$. The $\mathrm{MIC}$ was determined as the minimum concentration of doxycycline that resulted in no change in color. All experiments were carried out in triplicate. 


\section{Exposure to Static Antibiotic Concentrations}

We determined the effect of doxycycline against M. hyopneumoniae at static drug concentrations. The doxycycline concentrations used were $0,0.5,1,2,4,6,8,16,32$, and $64 \mathrm{MIC}$. Then, $0.4 \mathrm{ml}$ of the M. hyopneumoniae suspension in the exponential phase, 0.1 $\mathrm{ml}$ of drug solution, and $3.5 \mathrm{ml}$ of culture medium were added to a $7-\mathrm{ml}$ penicillin bottle. The initial inoculum size was $\sim 10^{6}$ $\mathrm{CFU} / \mathrm{ml}$. A growth control (M. hyopneumoniae culture without antimicrobial agents) and sterility control ( $4 \mathrm{ml}$ of blank medium) were included. Cultures were incubated at $37^{\circ} \mathrm{C}$ in an atmosphere of $5 \% \mathrm{CO}_{2}$ for $48 \mathrm{~h}$, and $100 \mu \mathrm{l}$ of each culture was collected at 0,1 , $3,6,9,12,24,36$, and 48 h for M. hyopneumoniae counting. Each sample was serially diluted 10 -fold, then 10 - $\mu$ l dilutions were transferred to agar plates. The latter were incubated for 7 days at $37^{\circ} \mathrm{C}$ in a humidified incubator in an atmosphere of $5 \% \mathrm{CO}_{2}$. Then, the number of $M$. hyopneumoniae colonies was recorded using an inverted microscope (Leica, Germany). The limit of detection was $100 \mathrm{CFU} / \mathrm{ml}$. Time-kill studies were repeated at least thrice on different days.

\section{Static Time-Kill Curves Fitting and Analysis}

The kill rate can reflect the effect of antibacterial drugs against a pathogen. The greater the growth rate, the stronger the antibacterial effect of the drug. The time-kill curves of doxycycline against $M$. hyopneumoniae were presented by plotting $\log _{10} \mathrm{CFU} /$ $\mathrm{ml}$ against time $(\mathrm{h})$ at different concentrations. First, the kill rates of $0-24 \mathrm{~h}, 0-36 \mathrm{~h}$, and $0-48 \mathrm{~h}$ time periods were calculated. The kill rates of 3-24 h, 3-36 h, and 3-48 h were also calculated because that a recovery growth was observed after transferring to the new culture vessel. Besides, the kill rates were also calculated from 12 and $48 \mathrm{~h}$ because there was no significant reduction in $M$. hyopneumoniae counts within $12 \mathrm{~h}$. Thus, eventually, the mean kill rate for seven time intervals (0-24 h, 0-36 h, 0-48 h, 3-24 h, $3-36 \mathrm{~h}, 3-48 \mathrm{~h}$, and $12-48 \mathrm{~h}$ ) was calculated by linear regression. The mean kill rate for these seven time intervals was selected to fit the drug concentration using the $E_{\max }$ model employing WinNonlin 6.1 (Pharsight, Mountain View, Sunnyvale, CA, USA). The $E_{\max }$ model can be presented as follows:

$$
E=\frac{E_{\max } \times C_{e}^{N}}{\mathrm{EC}_{50}^{N}+C_{e}^{N}}
$$

where $E$ is the kill rate, $E_{\max }$ is the maximum value of kill rate in a certain time interval, $C_{\mathrm{e}}$ is the doxycycline concentration, $N$ is the Hill coefficient (which describes the steepness of the kill rate-concentration curve), $\mathrm{EC}_{50}$ is the concentration at which $50 \%$ of the maximum kill rate is reached, and $R^{2}$ is the correlation coefficient of the relationship between experimental values and predicted values.

\section{Description of the In Vitro Dynamic Model}

According to the in vitro dynamic model previously established in our laboratory (Zhang et al., 2016; Zhang et al., 2018b; Huang et al., 2019). The study adopted a one-compartment open model with first-order absorption established by Huang et al. (2019) (Figure 2). This model was used to simulate the PK of doxycycline in porcine plasma (absorption half-life of $4.15 \mathrm{~h}$, elimination time of 5.9 h) (Bousquet et al., 1998). The model contained four compartments: an absorption chamber, a central chamber, a reserve chamber, and a recycle chamber. The model is based on an absorption chamber to simulate absorption and a central chamber in which the drug works. The absorption chamber acted as administration site, which contained $210 \mathrm{ml}$ sterile medium. The central chamber comprised $300 \mathrm{ml}$ of sterile medium (external compartment) and a $10-\mathrm{ml}$ volume dialysis tube (internal compartment) to culture bacteria. A reserve chamber was used to provide fresh medium, and an empty vessel was employed to collect waste. The compartments were connected by a fiberglass tube. Peristaltic pumps were used to operate the model, and the flowrate of the peristaltic pumps was set at 0.58 $\mathrm{ml} / \mathrm{min}$. Stirring hot plates were employed to mix the drug and provide a growth temperature $\left(37^{\circ} \mathrm{C}\right)$ for M. hyopneumoniae.

\section{Exposure to Dynamic Antibiotic Concentrations}

Ten milliliters of inoculum of turbidity $10^{7} \mathrm{CFU} / \mathrm{ml}$ was administered to the central dialysis tube. After M. hyopneumoniae had adapted to the new growth environment, and it was determined that the entire device was not contaminated; a certain drug dose was administered to the absorption chamber: this time was regarded as the initial time of the experiment. According to the clinically recommended dose, eight dose groups $(1,2.5,5,7.5,10,15,20$, and $30 \mathrm{mg} / \mathrm{kg}$ body weight) were designed in the in vitro dynamic model once daily for 3 days. Sampling was done $0 \mathrm{~h}$ before administration as well as $1,2,4$, $6,12,24,30,36,48,54,60,72,84$, and $96 \mathrm{~h}$ after administration for determination of the drug concentration). in addition, 0.1 $\mathrm{ml}$ of inoculum in the dialysis tube was sampled $0 \mathrm{~h}$ before administration as well as $6,12,24,30,36,48,54,60,72,84$, and $96 \mathrm{~h}$ after administration for bacterial counting. All experiments were carried out in triplicate.

\section{Susceptibility Testing of $\boldsymbol{M}$. hyopneumoniae}

The samples were collected at 24, 48, 72, and $96 \mathrm{~h}$ from a dialysis tube, which were cultured to the logarithmic growth phase. Then, the exponential-phase culture was diluted to the inoculum size of $10^{6} \mathrm{CFU} / \mathrm{ml}$, and $10 \mu \mathrm{l}$ of dilution was dropped on the surface of drug plates that contained 1 MIC concentration. After 7 days of culture, the colonies recovered on the plate were inoculated into the liquid medium for further culture. After continuous passaging for five times, the MIC of the strain was determined by a microdilution method. If the MIC value of the strain remained high, it indicates that the strain reduced sensitivity.

\section{Intergration and Modeling of PK/PD}

$\mathrm{PK} / \mathrm{PD}$ parameters were derived from time-concentration assays, and the antimycoplasmal effect was obtained from dynamic time-kill curves. The area under the concentration-time curve at $24 \mathrm{~h}\left(\mathrm{AUC}_{24 \mathrm{~h}}\right)$ was calculated using the trapezoidal method, and the $C_{\max }$ after each drug administration was observed directly from the curve. The parameter of $\% \mathrm{~T}>\mathrm{MIC}$ were calculated 
according to a noncompartmental analysis by WinNonlin. Then, data were analyzed using the sigmoid $E_{\max }$ model as follows:

$$
E=E_{\max }-\frac{\left(E_{\max }-E_{0}\right) \times C_{e}^{N}}{\mathrm{EC}_{50}^{N}+C_{e}^{N}}
$$

where $E$ is the antimycoplasmal effect; $E_{\max }$ is the change of control sample $\left(\log _{10} \mathrm{CFU} / \mathrm{ml}\right)$ in periods of $0-24 \mathrm{~h}$ after each administration; $E_{0}$ is the maximum value of the antimycoplasmal effect; $C_{\mathrm{e}}$ is the value of $\mathrm{PK} / \mathrm{PD}$ indices $\left(C_{\max } / \mathrm{MIC}, \mathrm{AUC}_{24 \mathrm{~h}} / \mathrm{MIC}\right.$ and $\left.\% \mathrm{~T}>\mathrm{MIC}\right) ; \mathrm{EC}_{50}$ is the $\mathrm{PK} / \mathrm{PD}$ index that produces a $50 \%$ reduction of the maximum antimycoplasmal effect; and $N$ is the Hill coefficient.

\section{RESULTS}

\section{Minimum Inhibitory Concentration}

The MIC of doxycycline against M. hyopneumoniae ATCC25934 as determined by the microdilution method was $0.125 \mu \mathrm{g} / \mathrm{ml}$.

\section{Static Time-Kill Curves and Analysis}

The static time-kill curve is shown in Figure 1: doxycycline produced a marked reduction in the range $0.41-2.76 \log _{10} \mathrm{CFU} / \mathrm{ml}$ within $48 \mathrm{~h}$. When the drug concentration was $<2$ MIC, mild inhibition of $M$. hyopneumoniae proliferation compared with that in the growth control group was observed, and M. hyopneumoniae showed a slow increase relative to the initial inoculum size. When the drug concentration was $>4 \mathrm{MIC}$, doxycycline showed bacteriostatic activity against $M$. hyopneumoniae within $48 \mathrm{~h}$ but did not show a bactericidal effect at all doses. There was no obvious change in the number of $M$. hyopneumoniae colonies within $12 \mathrm{~h}$, except for a decrease at $<64$ MIC, which had a reduction of $0.39 \log _{10} \mathrm{CFU} / \mathrm{ml}$. Figure 1 shows that a higher concentration of antibiotic elicited a better antibacterial effect.

The relationship between the kill rate and concentration is presented in Figure 2 (time interval was $12-48 \mathrm{~h}$ ). The $R^{2}$ estimated from the different time intervals ranged from 0.970 to 0.986 . The maximum $R^{2}(0.986)$ arose during the period $12-48 \mathrm{~h}$, and the maximum kill rate was $0.11 / \mathrm{h}$. The profile of the kill rate demonstrated that, if the concentration was $\leq 16$ MIC, the kill rate increased with increasing concentration. The relationship between concentration and kill rate was fitted by the $E_{\max }$ model, and the obtained parameters of $E_{\max }, \mathrm{EC}_{50}$, and the Hill coefficient are presented in Table $\mathbf{1 .}$

\section{Pharmacokinetics in the In Vitro Dynamic Model}

The concentration-time curve of the in vitro dynamic model for doxycycline is displayed in Figure 3: the peak concentration was reached $\sim 6 \mathrm{~h}$ after each doxycycline administration. $\mathrm{AUC}_{24 \mathrm{~h}}$ and $C_{\max }$ after each drug administration was $1.78-48.4 \mu \mathrm{g} \mathrm{h} / \mathrm{ml}$ and $0.16-3.41 \mu \mathrm{g} / \mathrm{ml}$, respectively. According to the recommended dose of $10 \mathrm{mg} / \mathrm{kg}$ body weight, the peak concentration could reach $1.49 \mu \mathrm{g} / \mathrm{ml}$ in a single administration, which is well above the MIC $(0.125 \mu \mathrm{g} / \mathrm{ml})$ of doxycycline against $M$. hyopneumoniae. Data were analyzed according to a one-compartmental model by WinNonlin and are shown in Figure 4. The concentrationtime profile of doxycycline in the in vitro dynamic model fitted a one-compartment open model with first-order absorption $\left(R^{2}=0.994\right)$.

\section{Pharmacokinetic/Pharmacodynamic Modeling and Analysis}

Figure 5 shows the antibacterial efficacy of doxycycline against $M$. hyopneumoniae at eight simulated dosing regimens. The reduction in bacterium $\left(\log _{10} \mathrm{CFU} / \mathrm{ml}\right)$ for several dosing regimens $(1,2.5,5$, $7.5,10,15,20$, and $30 \mathrm{mg} / \mathrm{kg}$ body weight) was $0.16,1.29,1.75$, $2.94,3.35,3.91,4.35$, and 5.77 during the entire experiment, respectively. Within the interaction at $24 \mathrm{~h}$, there was no marked decrease in the maximum reduction $\left(0.31 \log _{10} \mathrm{CFU} / \mathrm{ml}\right)$. When the dose was $<7.5 \mathrm{mg} / \mathrm{kg}$ body weight, doxycycline could kill $M$. hyopneumoniae (maximum reduction $=2.94 \log _{10} \mathrm{CFU} / \mathrm{ml}$ ). When the dose was $>10 \mathrm{mg} / \mathrm{kg}$ body weight, continuous administration for 3 days could achieve a bactericidal effect. At $30 \mathrm{mg} / \mathrm{kg}$ body weight, the number of $M$. hyopneumoniae could fall below the

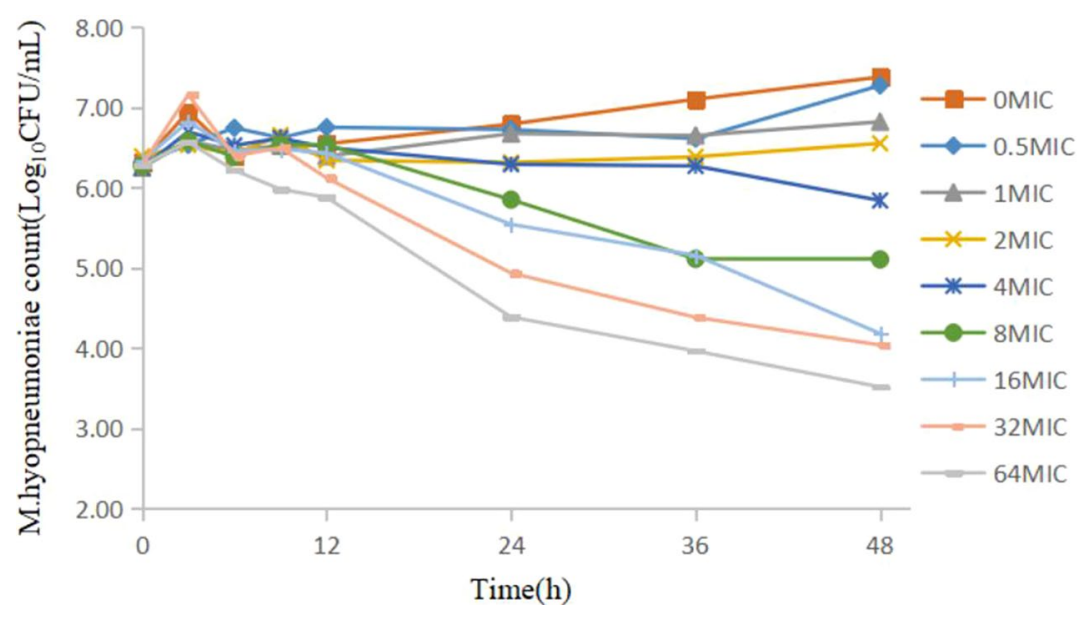

FIGURE 1| Static time-kill curve of doxycycline against Mycoplasma hyopneumoniae at different concentrations. 


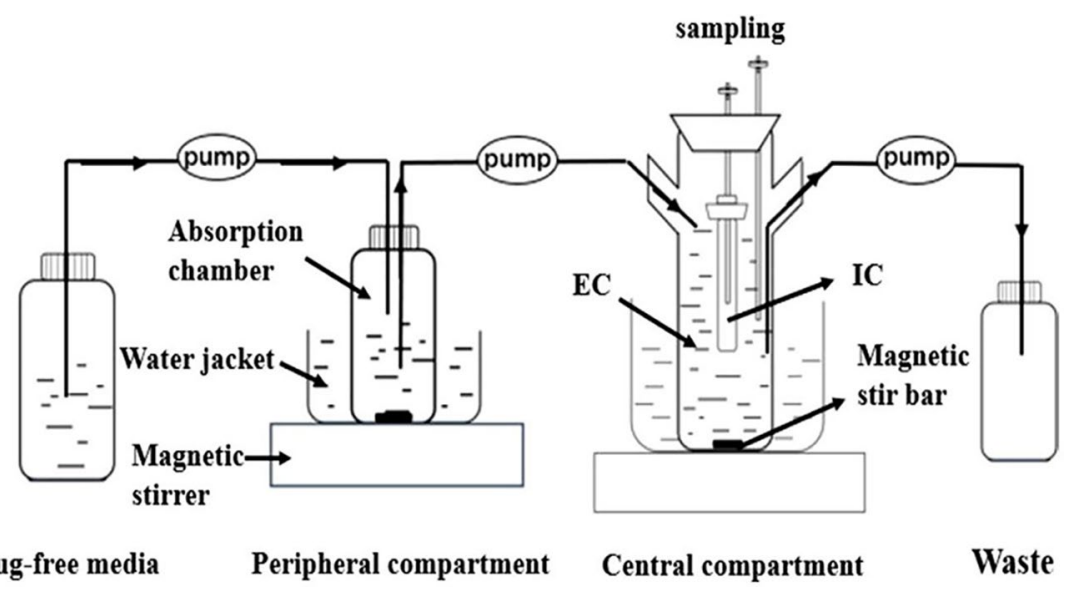

FIGURE 2 | The in vitro model that simulates the pharmacokinetics of doxycycline and determines a drug's effect on the growth and susceptibility of $M$. hyopneumoniae.

TABLE 1 | The kill rate parameter estimation derived from the $E_{\max }$ model.

\begin{tabular}{lcccc}
\hline Time (h) & $\boldsymbol{E}_{\max }\left(\mathbf{h}^{-1}\right)$ & $\mathbf{E C}_{\mathbf{5 0}}(\boldsymbol{\mu g} / \mathbf{m l})$ & Hill's slope & $\boldsymbol{R}^{\mathbf{2}}$ \\
\hline 0-24 & 0.09 & 2.82 & 1.73 & 0.974 \\
$0-36$ & 0.07 & 1.62 & 1.75 & 0.970 \\
$0-48$ & 0.05 & 1.09 & 2.53 & 0.975 \\
3-24 & 0.24 & 13.04 & 0.78 & 0.947 \\
3-36 & 0.09 & 1.18 & 1.74 & 0.985 \\
3-48 & 0.07 & 0.98 & 2.2 & 0.985 \\
12-48 & 0.06 & 0.78 & 2.98 & 0.986
\end{tabular}

$E_{\text {max }}$, the maximum value of kill rate in a certain time interval; $E C_{50}$, the concentration at which $50 \%$ of the maximum kill rate is reached; $R^{2}$, the correlation coefficient of the relationship between experimental values and predicted values. limit of detection $(100 \mathrm{CFU} / \mathrm{ml})$ during the entire experiment. Hence, doxycycline had a significant effect on M. hyopneumoniae, and escalating doses of doxycycline resulted in marked killing of M. hyopneumoniae. There have no drug-resistant strains been obtained under various dosing regimens.

The $E_{\max }$ relationships of three $\mathrm{PK} / \mathrm{PD}$ parameters $\left(\mathrm{AUC}_{24 \mathrm{~h}} /\right.$ MIC, $C_{\max } / \mathrm{MIC}, \mathrm{T}>\mathrm{MIC}$ ) versus the antimycoplasmal effect are shown in Figure 6. The $R^{2}$ of $\mathrm{AUC}_{24 \mathrm{~h}} / \mathrm{MIC}, C_{\max } / \mathrm{MIC}$, and $\% \mathrm{~T}>$ MIC with an antimycoplasmal effect was $0.917,0.923$, and 0.823 , respectively. These results suggested that the effect of doxycycline against M. hyopneumoniae was concentration dependent and that efficacy was driven by $\mathrm{AUC}_{24 \mathrm{~h}} / \mathrm{MIC}$ and $C_{\max } / \mathrm{MIC}$. The relationship between antibacterial efficacy and $\mathrm{PK} / \mathrm{PD}$ parameters was assessed by a sigmoid $E_{\max }$ model, and the obtained

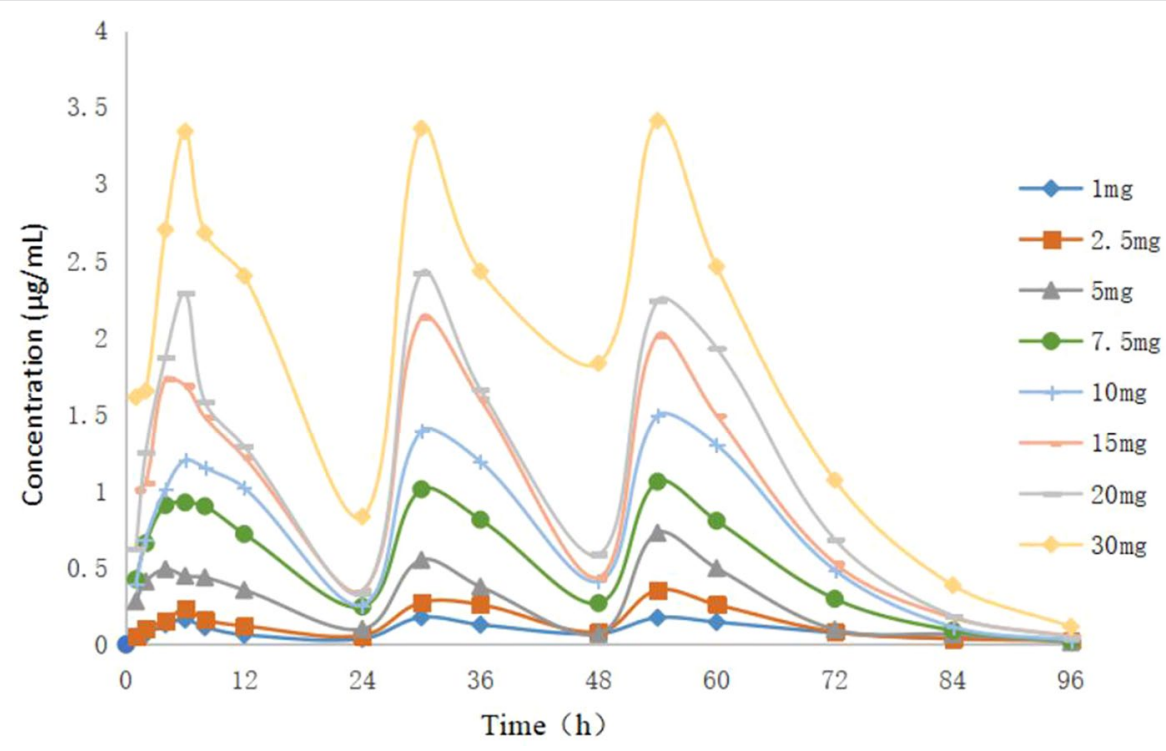

FIGURE 3 | Concentration-time curve of doxycycline in the dynamic mode. 


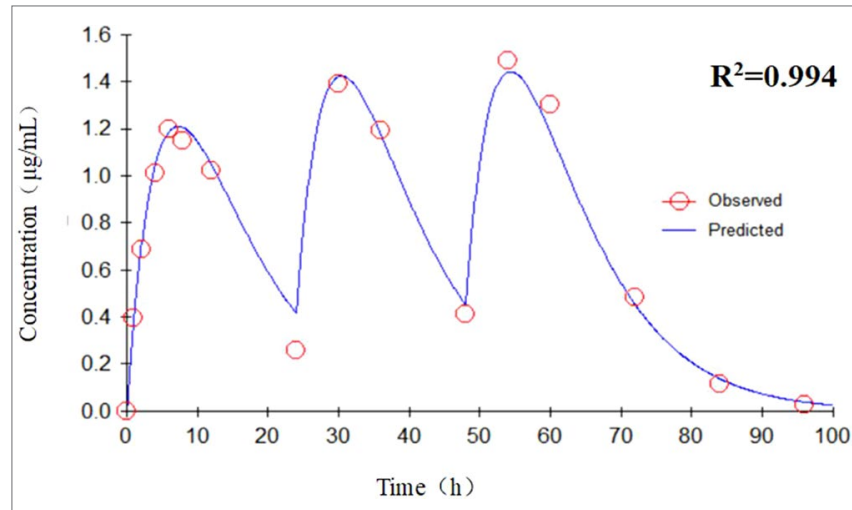

FIGURE 4 | The concentration-time profile fitted to a one-compartment open model with first-order absorption by WinNonlin.

parameters of $E_{0}, E_{\max }$, and $\mathrm{EC}_{50}$, and Hill coefficient are presented in Table 2. The $\mathrm{AUC}_{24 \mathrm{~h}} / \mathrm{MIC}$ and $C_{\max } / \mathrm{MIC}$ for producing a drop in $\log _{10} \mathrm{CFU} / \mathrm{ml}$ of 1 was $164 \mathrm{~h}$ and 9.89 , respectively.

$\mathrm{AUC}_{24 \mathrm{~h}} / \mathrm{MIC}$, the area under the curve at $24 \mathrm{~h}$ by MIC; $C_{\max } / \mathrm{MIC}$, peak concentration by MIC; $E_{\max }$, the change of control sample $\left(\log _{10} \mathrm{CFU} / \mathrm{ml}\right)$ in periods of $0-24 \mathrm{~h}$ after each administration; $E_{0}$, the maximum value of the antimycoplasmal effect; $\mathrm{EC}_{50}$, the $\mathrm{PK} / \mathrm{PD}$ index that produces a $50 \%$ reduction of the maximum antimycoplasmal effect.

\section{DISCUSSION}

In vitro dynamic models provide conditions for the studying of PK/PD of mycoplasma. M. hyopneumoniae is an important infectious pathogen in pigs and causes major economic losses to the pig industry. However, relevant studies have been hindered by three main difficulties. First, the characteristics of Mycoplasma species (small genome, small volume, and absence of a cell wall) mean that the nutrients and environment for in vitro culture must be controlled strictly (Beier and Siqueira, 2018). Some Mycoplasma strains can be cultured in a rich and complex medium supplemented with a large amount of serum, but the turbidity in media limits research. Second, isolating M. hyopneumoniae is very difficult because of its slow growth (and potential overgrowth) with other Mycoplasma species (Maes et al., 2018). Third, M. hyopneumoniae infection is complicated, lengthy, and affected by strain diversity, virulence, and host autoimmunity, which makes establishment of animal models difficult (Razin and Jacobs, 1992). Limited information is available on the PK/PD interactions of antibacterial agents against Mycoplasma species using in vitro or animal PK/PD models. In the present study, a one-compartment open model with first-order absorption was adopted and was used to simulate the PK of doxycycline in porcine plasma.

This PK/PD model reflects the relationship between the drug, bacteria, and quantification of the activity (as well as the likely efficacy) of antimicrobial agents against target pathogens (Zhang et al., 2018a). The dynamic model that we used provided a continuous flow of doxycycline to simulate the PK of doxycycline in pigs. In addition, a dialysis tube was used to culture bacteria, which (i) provided a steady source of nutrients for M. hyopneumoniae and (ii) avoided loss of M. hyopneumoniae. Samples could be collected continuously to monitor the growth and death of M. hyopneumoniae.

The PK/PD model used in the study is functionally superior to other models. Compared with the older dynamic model using only the central effect chamber, this model adds an absorption chamber to simulate drug absorption. Bacteria were cultured in dialysis tubes that allowed exchange of drug and nutrients (Meletiadis et al., 2012). We counted M. hyopneumoniae colonies on agar plates to investigate the effect of doxycycline against $M$. hyopneumoniae. The in vitro dynamic time-kill curve could characterize the activity of doxycycline against $M$. hyopneumoniae preliminarily. Where appropriate, data were fitted to the sigmoid $E_{\max }$ model to obtain specific $\mathrm{PK} / \mathrm{PD}$ parameters, and the PK/

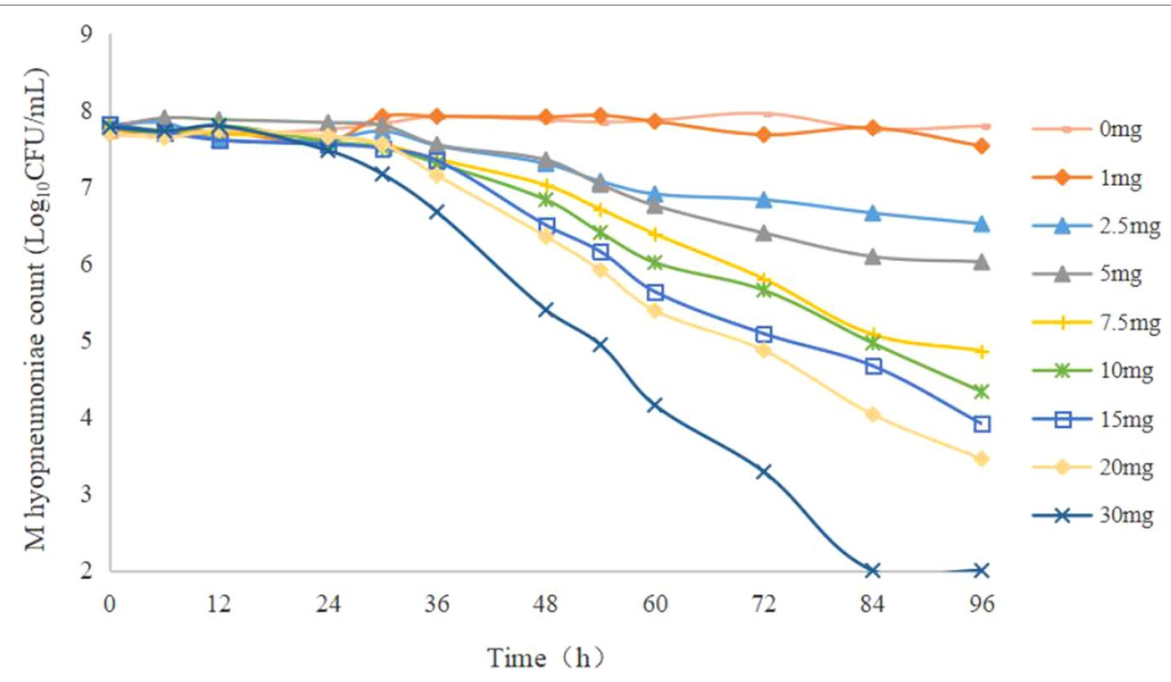

FIGURE 5 | Dynamic time-kill curve at different simulated doses. 

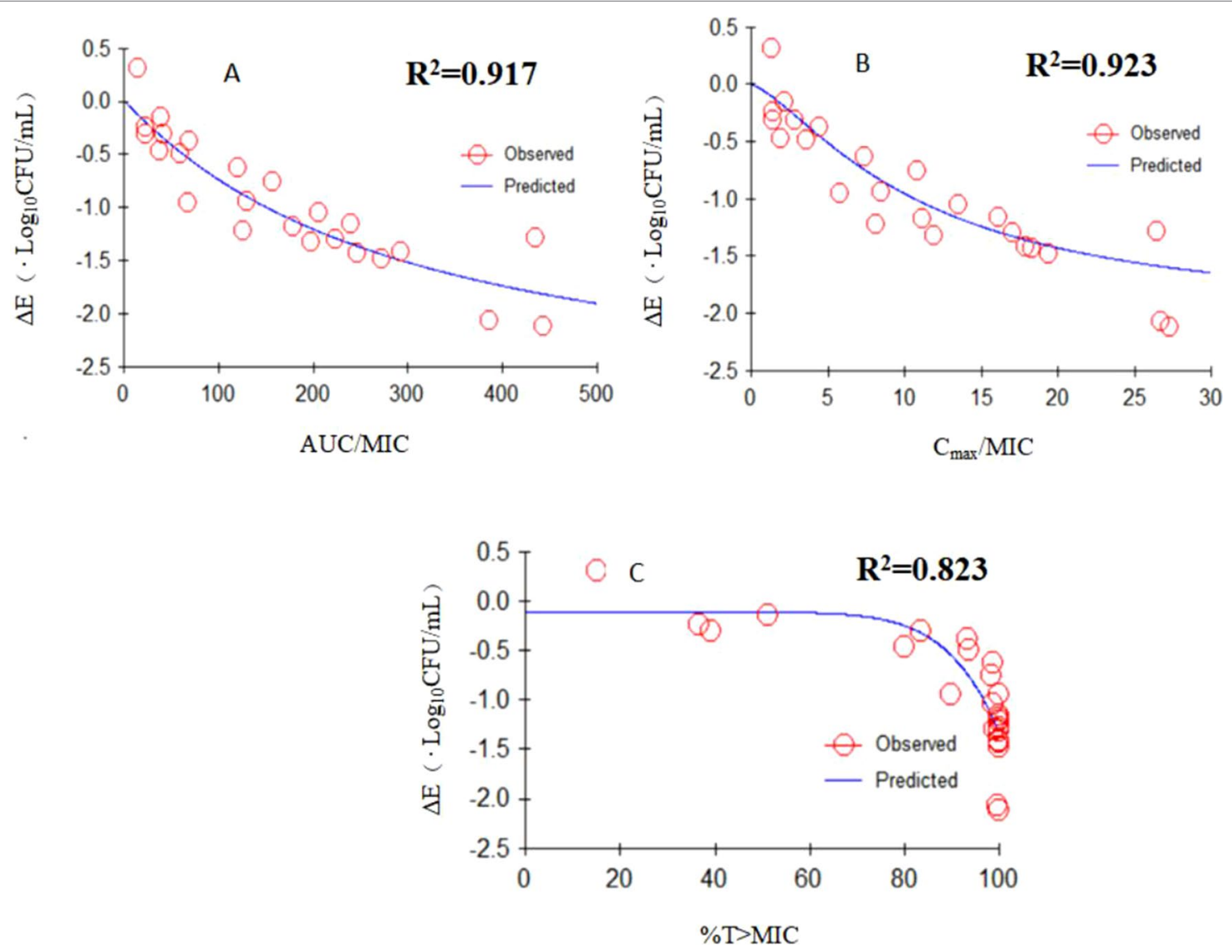

FIGURE $6 \mid E_{\max }$ relationships for the three PK/PD parameters versus antimycoplasmal effect. (A) $\mathrm{AUC}{ }_{24} \mathrm{~h} / \mathrm{MIC}$; (B) $C_{\max } / \mathrm{MIC}$; (C) $T>\mathrm{MIC}$.

TABLE 2 | The kill rate parameter estimation derived from the $E_{\max }$ model.

\begin{tabular}{lcc}
\hline PK/PD parameter & AUC $_{\mathbf{2 4}}$ /MIC (h) & $\boldsymbol{C}_{\text {max }} / \mathbf{M I C}$ \\
\hline$E_{\max }\left(\log _{10} \mathrm{CFU} / \mathrm{ml}\right)$ & $-3 \times 10^{-05}$ & $-5 \times 10^{-05}$ \\
$\mathrm{EC}_{50}$ & 179 & 21 \\
$E_{0}\left(\log _{10} \mathrm{CFU} / \mathrm{ml}\right)$ & -2.13 & -3.14 \\
Hill's slope & 1.38 & 1.01 \\
$R^{2}$ & 0.917 & 0.923 \\
$1 \log _{10} \mathrm{CFU} / \mathrm{ml}$ drop & $164 \mathrm{~h}$ & 9.89 \\
\hline
\end{tabular}

PD index obtained was used to calculate the parameter value corresponding to a certain antibacterial effect. We could investigate the PK/PD interactions of doxycycline against M. hyopneumoniae to determine the concentration and/or time dependence of such activity and provide guidance for designing dose regimens for the clinical treatment of $M$. hyopneumoniae infection.

Referring to relevant study reports, the value of MIC is within reasonable range. The MIC for doxycycline against M. hyopneumoniae determined using a microdilution method was $0.125 \mu \mathrm{g} / \mathrm{ml}$. To be consistent with the in vitro experiment of time-kill curves, the final inoculum of M. hyopneumoniae in each well was $10^{6} \mathrm{CFU} / \mathrm{ml}$ in our study. The recommended turbidity of MIC testing against veterinary Mycoplasma species is $10^{3}-10^{5} \mathrm{CFU} / \mathrm{ml}$ (Hannan, 2000). The turbidity of the growth phase of M. hyopneumoniae seems to be not so important, and the inoculum size exhibits little effect on the MIC determination of Mycoplasma species. The test strain ATCC25934 is a standard strain of M. hyopneumoniae, and its antibiotic sensitivity is different in different clinical isolates to some extent. Felde et al. (2018) calculated the MIC value of doxycycline against 44 M. hyopneumoniae field strains isolated from Central Europe by a microbroth-dilution method. $\mathrm{MIC}_{50}$ (the minimal concentration that inhibits colony formation by $50 \%$ ) and $\mathrm{MIC}_{90}$ (the minimal concentration that inhibits colony formation by $90 \%$ ) was 0.078 and $0.312 \mu \mathrm{g} / \mathrm{ml}$, respectively. Moreover, most studies from Europe have demonstrated similar values of $\mathrm{MIC}_{50}$ and $\mathrm{MIC}_{90}$ for oxytetracycline (Tavio et al., 2014).

The study has shown that doxycycline has a significant effect on M. hyopneumoniae. Figure 1 shows the static time-kill curve. When the doxycycline concentration was $>4$ MIC, doxycycline showed bacteriostatic activity against $M$. hyopneumoniae within $48 \mathrm{~h}$ and produced a maximum reduction of $2.76 \log _{10} \mathrm{CFU} / \mathrm{ml}$ at $64 \mathrm{MIC}$. Figure 5 shows the antibacterial efficacy of doxycycline against M. hyopneumoniae in our dynamic PK/PD model. We found that doxycycline could kill $M$. hyopneumoniae. When the dose was $>10 \mathrm{mg} / \mathrm{kg}$ body weight, continuous interactions could result in a bactericidal effect during the experiment. At the dose of $30 \mathrm{mg} / \mathrm{kg}$ body weight, doxycycline produced a maximum antimycoplasmal effect of $5.77 \log _{10} \mathrm{CFU} / \mathrm{ml}$ reduction within $96 \mathrm{~h}$. Figure 5 indicated that escalating doses of doxycycline resulted in marked killing 
of $M$. hyopneumoniae. The results of $E_{\max }$ model verified that the antibacterial effect of doxycycline was concentration dependent, in which the relationship between antimycoplasmal effect and PK/PD parameters was fitted. The magnitude of $\mathrm{AUC}_{24 \mathrm{~h}} / \mathrm{MIC}$ and $C_{\max } /$ MIC predicted for $1 \log _{10}(\mathrm{CFU} / \mathrm{ml})$ reduction was $164 \mathrm{~h}$ and 9.89 , respectively. These data provided a scientific dosing guidance of doxycycline against $M$. hyopneumoniae.

There have no drug-resistant strains been obtained in this study. We tried to screen for drug-resistant strains of M. hyopneumoniae in an in vitro dynamic model using drug-containing agar plates. No drug-resistant strains were obtained under various dosing regimens during short-term experimental studies. The result indicated that M. hyopneumoniae is not easy to get resistant for doxycycline.

Studies have shown that doxycycline does not have activity against MmmSC if the bacteria are exposed to the drug for 12 and $24 \mathrm{~h}$ at a constant concentration (Mitchell et al., 2013). Those findings are not in accordance with a study that indicated doxycycline had a marked effect on M. gallisepticum (Zhang et al., 2016). In this study, irrespective of whether a static or dynamic drug concentration was used, there was no significant reduction in $M$. hyopneumoniae counts within several hours of the start of the experiment. There could be three explanations for these results. First, delayed growth of $M$. hyopneumoniae was observed, and $M$. hyopneumoniae did not recover instantaneously in the logarithmic growth phase upon transfer to the new culture environment. Second, the antibacterial mechanism of doxycycline is to inhibit protein synthesis, and it acted as a long-acting bacteriostatic agent (Schmidt et al., 2018; Chukwudi and Good, 2019). Third, due to the slow growth of $M$. hyopneumoniae, bacteriostatic agents (e.g., doxycycline) need longer to elicit their effects.

According to the bactericidal properties of drug on the bacteria, drugs can be divided into time- and concentration-dependent drugs. When the antibiotic concentration is increased, concentrationdependent drugs lead to faster killing, whereas the kill rate of timedependent drugs might be remained constant (Craig, 1998). In this study, static time-killing and dynamic study both showed that a higher concentration of antibiotic elicited a better antibacterial effect. The results of dynamic time-killing study showed that the $C_{\max }$ /MIC parameter had the highest correlation with the antibacterial effects $\left(R^{2}=0.923\right)$. Therefore, we could infer that the antimicrobial effect of doxycycline against $M$. hyopneumoniae was concentration dependent. In this study, WinNonlin was employed to fit the drug concentration and kill rate (obtained from different intervals). The results suggest that higher doxycycline concentration result in faster kill rate until the doxycycline concentration was $>16$ MIC. In traditional PK/PD studies, bacteria are exposed to a

\section{REFERENCES}

Agwuh, K. N., and MacGowan, A. (2006). Pharmacokinetics and pharmacodynamics of the tetracyclines including glycylcyclines. J. Antimicrob. Chemother. 58, 256265. doi: $10.1093 / \mathrm{jac} / \mathrm{dkl} 224$

Baert, K., Croubels, S., Gasthuys, F., De Busser, J., and De Backer, P. (2000). Pharmacokinetics and oral bioavailability of a doxycycline formulation (DOXYCYCLINE 75\%) in nonfasted young pigs. J. Vet. Pharmacol. Ther. 23, 45-48. doi: 10.1046/j.1365-2885.2000.00235.x constant drug concentration, and efficacy evaluation is based on time-kill curves (Zhanel et al., 1991). This method can be used to study the initial kill rate of bacteria and the ability of bacterial regrowth. However, the drug concentrations in serum and target tissue are changing continuously, so the dynamic model used in our study is clinically important. The rapid killing observed in the in vitro dynamic time-kill curves suggested that doxycycline exhibited a concentration-dependent killing mechanism against $M$. hyopneumoniae. Data were fitted to a sigmoidal $E_{\max }$ model, and the PK/PD parameters that correlated most closely to efficacy were $\mathrm{AUC}_{24 \mathrm{~h}} / \mathrm{MIC}\left(R^{2}=0.917\right)$ and $C_{\max } / \mathrm{MIC}\left(R^{2}=0.923\right)$. Doxycycline seemed to be driven by the AUC.

However, this result is not in complete accordance with the literatures that have been made about the PDs of doxycycline. The effect of doxycycline on Gram-positive pathogens (e.g., Staphyloccus aureus, Steptococcus pneumonia) and Gram-negative pathogens (e.g., Pasteurella multocida, Escherichia coli) suggests that doxycycline kills bacteria by time-dependent kinetics at low concentrations but by concentration-dependent kinetics at high concentrations (Cunha et al., 2000). In a study of the effects of doxycycline against M. gallisepticum, the time-kill curves showed time-dependent characteristics (Zhang et al., 2016). Hence, at present, this area is understood incompletely, and our study will aid understanding (Agwuh and MacGowan, 2006). In addition, the concentrationdependent characteristics must be confirmed by further studies using an in vivo PK/PD model and other Mycoplasma strains.

\section{DATA AVAILABILITY}

The datasets generated for this study are available on request to the corresponding author.

\section{AUTHOR CONTRIBUTIONS}

$\mathrm{HD}$ and $\mathrm{HZ}$ conceived and designed the experiments. HZ, $\mathrm{CM}$, and JL performed the experiments. $\mathrm{HZ}$ and $\mathrm{ZH}$ analyzed data and wrote the manuscript. XG, XS, and HD revised the manuscript and supervision of the entire project. All authors read and approved the final manuscript.

\section{FUNDING}

This work was supported by the National Key Research and Development Program of China (grant numbers 2016YFD0501300 and 2016YFD0501310). 
Castro, L. J., Sahagun, A. M., Diez, M. J., Fernandez, N., Sierra, M., and Garcia, J. J. (2009). Pharmacokinetics of doxycycline in sheep after intravenous and oral administration. Vet. J. 180, 389-395. doi: 10.1016/j.tvjl.2008.02.001

Castro Robles, L. J., Sahagun Prieto, A. M., Diez Liebana, M. J., Fernandez Martinez, N., Sierra Vega, M., and Garcia Vieitez, J. J. (2012). Pharmacokinetic behavior of doxycycline after intramuscular injection in sheep. Am. J. Vet. Res. 73, 714-718. doi: 10.2460/ajvr.73.5.714

Chukwudi, C. U., and Good, L. (2019). Doxycycline inhibits pre-rRNA processing and mature rRNA formation in E. coli. J. Antibiot. 72, 225-236. doi: 10.1038/ s41429-019-0149-0

Craig, W. A. (1998). Pharmacokinetic/pharmacodynamic parameters: rationale for antibacterial dosing of mice and men. Clin. Infect. Dis. 26, 1-10; quiz 11-12. doi: $10.1086 / 516284$

Cunha,B.A.,Domenico,P., andCunha,C.B.(2000).Pharmacodynamicsofdoxycycline. Clin. Microbiol. Infect. 6, 270-273. doi: 10.1046/j.1469-0691.2000.00058-2.x

de la Pena, A., Grabe, A., Rand, K. H., Rehak, E., Gross, J., Thyroff-Friesinger, U., et al. (2004). PK-PD modelling of the effect of cefaclor on four different bacterial strains. Int. J. Antimicrob. Agents 23, 218-225. doi: 10.1016/j. ijantimicag.2003.07.009

De Mil, T., Devreese, M., De Saeger, S., Eeckhout, M., De Backer, P., and Croubels, S. (2016). Influence of mycotoxin binders on the oral bioavailability of doxycycline in pigs. J. Agric. Food Chem. 64, 2120-2126. doi: 10.1021/acs. jafc.5b06084

Felde, O., Kreizinger, Z., Sulyok, K. M., Hrivnak, V., Kiss, K., Jerzsele, A., et al. (2018). Antibiotic susceptibility testing of Mycoplasma hyopneumoniae field isolates from Central Europe for fifteen antibiotics by microbroth dilution method. PLoS One 13, e0209030. doi: 10.1371/journal.pone.0209030

Hannan, P. C. (2000). Guidelines and recommendations for antimicrobial minimum inhibitory concentration (MIC) testing against veterinary mycoplasma species. International Research Programme on Comparative Mycoplasmology. Vet. Res. 31, 373-395. doi: 10.1051/vetres:2000100

Hannan, P. C., Windsor, G. D., de Jong, A., Schmeer, N., and Stegemann, M. (1997). Comparative susceptibilities of various animal-pathogenic mycoplasmas to fluoroquinolones. Antimicrob. Agents Chemother. 41, 2037-2040. doi: 10.1051/ vetres: 2000100

Hartmann, A., Krebber, R., Daube, G., and Hartmann, K. (2008). Pharmacokinetics of pradofloxacin and doxycycline in serum, saliva, and tear fluid of cats after oral administration. J. Vet. Pharmacol. Ther. 31, 87-94. doi: 10.1111/j.1365-2885.2007.00932.x

Huang, Z., Wu, Y., Zhou, Z., Xia, X., Gu, X., Cai, Q., et al. (2019). Pharmacokinetic and pharmacodynamic integration and resistance analysis of tilmicosin against Mycoplasma gallisepticum in an in vitro dynamic model. Front. Pharmacol. 10, 670. doi: 10.3389/fphar.2019.00670

Li, X., Xie, S., Pan, Y., Qu, W., Tao, Y., Chen, D., et al. (2016). Preparation, characterization and pharmacokinetics of doxycycline hydrochloride and florfenicol polyvinylpyrroliddone microparticle entrapped with hydroxypropylbeta-cyclodextrin inclusion complexes suspension. Colloids Surf. B Biointerfaces 141, 634-642. doi: 10.1016/j.colsurfb.2016.02.027

Liu, T. (2015). Clinical trial of long-acting injection of doxycycline hydrochloride on swine enzootic pneumonia. Chin. Anim. Husb. Vet. Abstr. 31:225. (In Chinese).

Maes, D., Segales, J., Meyns, T., Sibila, M., Pieters, M., and Haesebrouck, F. (2008). Control of Mycoplasma hyopneumoniae infections in pigs. Vet. Microbiol. 126, 297-309. doi: 10.1016/j.vetmic.2007.09.008

Maes, D., Sibila, M., Kuhnert, P., and Segales, J. (2018). Update on Mycoplasma hyopneumoniae infections in pigs: knowledge gaps for improved disease control. Transbound. Emerg. Dis. 65, 110-124. doi: 10.1111/tbed.12677

Meijer, L. A., Ceyssens, K. G., de Greve, B. I., and de Bruijn, W. (1993). Pharmacokinetics and bioavailability of doxycycline hyclate after oral administration in calves. Vet. Q. 15, 1-5. doi: 10.1080/01652176.1993.9694358

Meletiadis, J., Al-Saigh, R., Velegraki, A., Walsh, T. J., Roilides, E., and Zerva, L. (2012). Pharmacodynamic effects of simulated standard doses of antifungal drugs against Aspergillus species in a new in vitro pharmacokinetic/pharmacodynamic model. Antimicrob. Agents Chemother. 56, 403-410. doi: 10.1128/AAC.00662-11

Mitchell, J. D., McKellar, Q. A., and McKeever, D. J. (2013). Evaluation of antimicrobial activity against Mycoplasma mycoides subsp mycoides small colony using an in vitro dynamic dilution pharmacokinetic/pharmacodynamic model. J. Med. Microbiol. 62, 56-61. doi: 10.1099/jmm.0.045971-0
Nicasio, A. M., Bulitta, J. B., Lodise, T. P., D’Hondt, R. E., Kulawy, R., Louie, A., et al. (2012). Evaluation of once-daily vancomycin against methicillin-resistant Staphylococcus aureus in a hollow-fiber infection model. Antimicrob. Agents Chemother. 56, 682-686. doi: 10.1128/AAC.05664-11

Razin, S., and Jacobs, E. (1992). Mycoplasma adhesion. Microbiology 138, 407-422. doi: 10.1099/00221287-138-3-407

Rebaque, F., Camacho, P., Parada, J., Lucchesi, P., Ambrogi, A., and Tamiozzo, P. (2018). Persistence of the same genetic type of Mycoplasma hyopneumoniae in a closed herd for at least two years. Rev. Argent. Microbiol. 50, 147-150. doi: 10.1016/j.ram.2017.05.002

Schmidt, K., Gardill, B. R., Kern, A., Kirchweger, P., and Borsch, M. (2018). Design of an allosterically modulated doxycycline and doxorubicin drugbinding protein. Proc. Natl. Acad. Sci. U. S. A. 115, 5744-5749. doi: 10.1073/ pnas. 1716666115

Sebbag, L., Showman, L., McDowell, E. M., Perera, A., and Mochel, J. P. (2018). Impact of flow rate, collection devices, and extraction methods on tear concentrations following oral administration of doxycycline in dogs and cats. J. Ocul. Pharmacol. Ther. 34, 452-459. doi: 10.1089/jop.2018.0008

Sibila, M., Martinez-Guino, L., Huerta, E., Llorens, A., Mora, M., Grau-Roma, L., et al. (2009). Swine torque teno virus (TTV) infection and excretion dynamics in conventional pig farms. Vet. Microbiol. 139, 213-218. doi: 10.1016/j. vetmic.2009.05.017

Tanner, A. C., Erickson, B. Z., and Ross, R. F. (1993). Adaptation of the Sensititre broth microdilution technique to antimicrobial susceptibility testing of Mycoplasma hyopneumoniae. Vet. Microbiol. 36, 301-306. doi: 10.1016/0378-1135(93)90096-P

Tavio, M. M., Poveda, C., Assuncao, P., Ramirez, A. S., and Poveda, J. B. (2014). In vitro activity of tylvalosin against Spanish field strains of Mycoplasma hyopneumoniae. Vet. Rec. 175, 538-U568. doi: 10.1136/vr.102458

Villarreal, I., Maes, D., Vranckx, K., Calus, D., Pasmans, F., and Haesebrouck, F. (2011). Effect of vaccination of pigs against experimental infection with high and low virulence Mycoplasma hyopneumoniae strains. Vaccine 29, 1731-1735. doi: 10.1016/j.vaccine.2011.01.002

Wang, S., He, X., and Zhao, Y. (2010). Clinical trial of long-acting injection of doxycycline hydrochloride on swine enzootic pneumonia. Guangdong Agric. Sci. 37, 186-188. doi: 10.16768/j.issn.1004-874x.2010.11.078 (In Chinese).

Weber Sde, S., Sant'Anna, F. H., and Schrank, I. S. (2012). Unveiling Mycoplasma hyopneumoniae promoters: sequence definition and genomic distribution. DNA Res. 19, 103-115. doi: 10.1093/dnares/dsr045

Yang, F., Yang, F., Wang, G., and Kong, T. (2018). Pharmacokinetics of doxycycline after oral administration of single and multiple dose in broiler chickens. J. Vet. Pharmacol. Ther. 41, 919-923. doi: 10.1111/jvp.12699

Zhanel, G. G., Karlowsky, J. A., Hoban, D. J., and Davidson, R. J. (1991). Antimicrobial activity of subinhibitory concentrations of aminoglycosides against Pseudomonas aeruginosa as determined by the killing-curve method and the postantibiotic effect. Chemotherapy 37, 114-121. doi: 10.1159/000238842

Zhang, L., Li, Y., Wang, Y., and Sajid, A. (2018a). Integration of pharmacokineticpharmacodynamic for dose optimization of doxycycline against Haemophilus parasuis in pigs. J. Vet. Pharmacol. Ther. 41, 706-718. doi: 10.1111/jvp.12512

Zhang, N., Gu, X., Ye, X., Wu, X., Zhang, B., Zhang, L., et al. (2016). The PK/PD interactions of doxycycline against Mycoplasma gallisepticum. Front. Microbiol. 7, 653-662. doi: 10.3389/fmicb.2016.00653

Zhang, N., Wu, Y., Huang, Z., Zhang, C., Zhang, L., Cai, Q., et al. (2018b). Relationship between danofloxacin $\mathrm{PK} / \mathrm{PD}$ parameters and emergence and mechanism of resistance of Mycoplasma gallisepticum in in vitro model. PLoS One 13, e0202070. doi: 10.1371/journal.pone.0202070

Conflict of Interest Statement: The authors declare that the research was conducted in the absence of any commercial or financial relationships that could be construed as a potential conflict of interest.

Copyright (C) 2019 Zhang, Mao, Li, Huang, Gu, Shen and Ding. This is an open-access article distributed under the terms of the Creative Commons Attribution License (CC $B Y)$. The use, distribution or reproduction in other forums is permitted, provided the original author(s) and the copyright owner(s) are credited and that the original publication in this journal is cited, in accordance with accepted academic practice. No use, distribution or reproduction is permitted which does not comply with these terms. 